\title{
INFLUENCE OF THE ELECTRIC DOUBLE LAYER ON INDUCED PRESSURE FIELDS AND DEVELOPMENT LENGTHS IN ELECTRO-OSMOTIC FLOWS
}

\author{
YONGHAO ZHANG*, XIAO-JUN GU, ROBERT W. BARBER AND DAVID R. EMERSON
}

\author{
Centre for Microfluidics and Microsystems Modelling, CCLRC Daresbury Laboratory, \\ Warrington, Cheshire, WA4 4AD, United Kingdom \\ *y.zhang@dl.ac.uk
}

\begin{abstract}
Electro-osmotic flow can be used as an efficient pumping mechanism in microfluidic devices. For this type of flow, frictional losses at the entrance and exit can induce an adverse longitudinal pressure distribution that can lead to dispersive effects. The present study describes a numerical investigation of the influence of the electric double layer on the induced pressure field and the flow development length. The induced pressure gradient is affected by the volumetric flow rate, fluid viscosity and the channel height. When the electric double layer is small, the development length remains constant at 0.57 of the channel height but decreases as the double layer grows in thickness.
\end{abstract}

Keywords: Microfluidics; electro-osmotic flow; induced pressure; entrance effects.

\section{Introduction}

Microfluidic devices that utilize electro-osmotic flow (EOF) have developed rapidly ${ }^{1-4}$ and are particularly suited to the production of many low-volume, high-value compounds used by the fine chemical and pharmaceutical industries. Compared to conventional devices, miniaturized systems offer a number of advantages that include reduced fabrication costs, shorter process times and reduced reagent consumption.

In an ideal EOF the velocity profile is plug-like and the mean velocity will be independent of the cross-sectional area provided the electric double layer (EDL) is much smaller than the characteristic length scale of the channel. As a consequence, dispersive effects are minimized and EOF offers an efficient method for manipulating fluids in miniaturized devices. However, electro-osmotic flows can be difficult to control due to the complex interactions arising from the surface composition, buffer characteristics, and the applied electric fields. Subtle hydrodynamic effects can also influence flow control.

Measuring flow properties in micro-devices, without disturbing the flow field, remains a challenging issue. Theory and simulation therefore have an important role to play in explaining many of the experimentally observed flow phenomena. For example, previous work ${ }^{5,6}$ demonstrated that the pressure losses associated with a sudden contraction (inlet) or expansion (outlet) would lead to an adverse pressure gradient being induced, as shown in Fig. 1. In the present work, a numerical study has been carried out to investigate the pressure changes at the ends of the channel and the flow development length. 


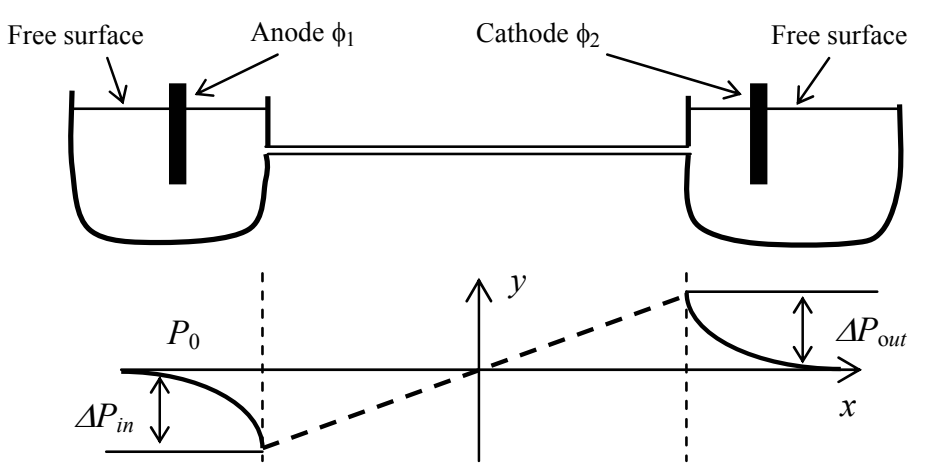

Fig. 1. Schematic diagram of an electro-osmotic flow in a micro-channel and the induced pressure field caused by inlet and outlet losses.

\section{Mathematical Model and Numerical Simulation}

Electro-osmotic flow can be modeled by adding a source term to the Navier-Stokes equations describing the electrical force acting on the ions. The governing equations can be found in the literature ${ }^{6,7}$ and are given by

$$
\begin{gathered}
\nabla \cdot \boldsymbol{u}=0, \\
\rho \frac{D \boldsymbol{u}}{D t}=-\nabla p+\mu \nabla^{2} \boldsymbol{u}+\boldsymbol{F},
\end{gathered}
$$

where $\boldsymbol{u}$ is the velocity vector, $\rho$ is the fluid density, $\mu$ is the viscosity and $p$ is the static pressure. The electrical force, $\boldsymbol{F}$, acting on the fluid is given by

$$
\boldsymbol{F}=-\rho_{e} \nabla(\psi+\phi)
$$

where $\rho_{e}$ is the electric charge density, $\psi$ is the electric potential due to the zeta potential at the wall, and $\phi$ is the applied electric field described by Laplace's equation, i.e. $\nabla^{2} \phi=0$. The local net charge density, $\rho_{e}$, can be determined from $\nabla^{2} \psi=-\rho_{e} / \varepsilon$, where $\varepsilon$ is the electric permittivity of the solution. This equation can be simplified by employing the Debye-Hückel approximation to give $\nabla^{2} \psi=k^{2} \psi$, where $k^{-1}$ is the Debye length which characterizes the thickness of the electric double layer. Further details and discussion of the present numerical model can be found in Ref. 7.

To minimize the influence of boundary errors, the present study has modeled the entire flow field (including the reservoirs). The boundaries of the upstream and downstream reservoirs were placed $20 d$ away from the channel, where $d$ is the height of the channel. Increasing the location of the boundary further had a negligible effect on the results. For ease of computation, the zeta potential along the surface of the channel was considered to be uniform and the reservoir levels were assumed to remain constant. In order to keep the computational costs at a realistic level, the flow simulations were 
performed in 2D which can be justified because practical electro-osmotic devices are often fabricated with high aspect ratios.

The electric double layer thickness can range from 1-100 nm, which is about 100 times smaller than the height of a typical micro-channel. To resolve the entire EDL is therefore computationally impractical. Instead, the Helmholtz-Smoluchowski slip boundary condition ${ }^{8}$ was applied at the edge of the EDL. The grid point next to the wall was assumed to be outside the EDL and the no-slip condition was applied at the wall.

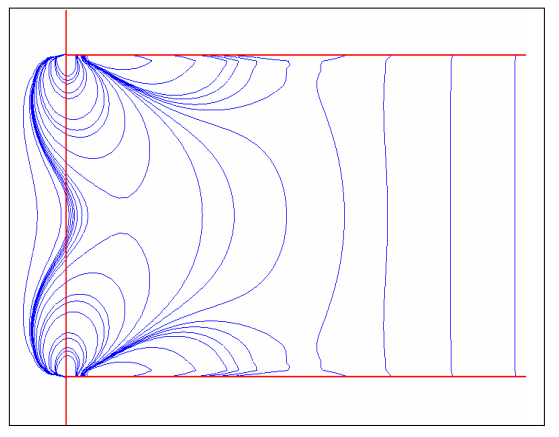

(a)

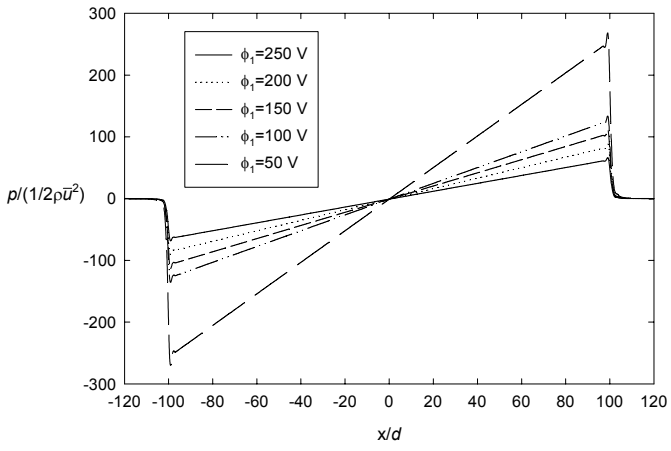

(b)

Fig. 2. a) pressure contours at the micro-channel inlet, $\phi_{1}-\phi_{2}=200 \mathrm{~V}$; b) longitudinal pressure distribution, $\phi_{2}=0 \mathrm{~V}$. Here, $d=10 \mu \mathrm{m}, k^{-1}=10 \mathrm{~nm}$, and $\bar{u}$ is the mean velocity in the channel.

In the present paper, the numerical simulations have used the commercial Computational Fluid Dynamics software, CFD-ACE+. ${ }^{9}$ The liquid was assumed to be a Newtonian aqueous solution with the following properties: dielectric constant, $\varepsilon_{\mathrm{r}}=\varepsilon /$ permittivity of free space $=78.5$, viscosity, $\mu=0.001 \mathrm{Nsm}^{-2}$, and zeta potential, $\zeta=-100 \mathrm{mV}$. In all computations, the channel length was taken to be $200 \mathrm{~d}$. The pressure contours shown in Fig. 2a illustrate that the flow develops very rapidly and the pressure quickly establishes a uniform profile across the channel. Figure $2 \mathrm{~b}$ shows the influence of the externally applied electric field on the induced pressure distribution. The simulations predict a sudden drop in pressure at the entrance followed by a gradual increase in pressure along the channel and another sudden drop at the exit. Electro-osmotic flow can therefore induce an adverse pressure gradient along the length of the channel which compensates for the energy losses at the entrance and exit. In contrast, simulations by Yang et al. ${ }^{10}$ have recently claimed that the induced pressure gradient is zero within the fully-developed region if the channel length is $>50 \mathrm{~d}$. A theoretical explanation was also proposed to support their findings but it contained an incorrect assumption that $\partial^{2} u / \partial y^{2}=0$ along the centerline of the channel. This boundary condition will always result in a zero pressure gradient in the fully-developed region. The reasons for the discrepancy between the results given by Yang et al. and the present work are unclear.

In the current analysis, the development length, $l_{d}$, is found to be independent of Reynolds number for creeping flow. We have defined $l_{d}$ as the distance from the inlet to the location where the fluid velocity along the centerline of the channel reaches $99 \%$ of 
the fully-developed value. For $k^{-1} d^{-1}$ less than $10^{-3}$, the flow development length, $l_{d}$, was found to be constant at $0.57 d$. Interestingly, the value of $l_{d}$ then decreases with increasing Debye layer (see Table 1). In comparison, $l_{d}$ is $0.63 d$ for pressure driven creeping flow. ${ }^{11}$

Table 1. Influence of the electric double layer on the flow development length, $l_{d}$.

\begin{tabular}{|c|c|c|c|c|c|c|c|c|c|c|}
\hline$k^{-1} d^{-1} \times 10^{3}$ & 0.02 & 0.1 & 0.2 & 0.5 & 1 & 2 & 3 & 4 & 5 & 10 \\
\hline$l_{d} / d$ & 0.57 & 0.57 & 0.57 & 0.57 & 0.56 & 0.55 & 0.53 & 0.53 & 0.52 & 0.42 \\
\hline
\end{tabular}

\section{Conclusions}

Electro-osmotic flow creates an induced pressure gradient as a result of pressure losses at the entrance and exit of the channel. In previous numerical simulations, this effect has either been ignored or misunderstood. The present simulations have shown that the induced pressure gradient depends on the volumetric flow rate, the fluid viscosity and the channel height. In addition, the thickness of the electric double layer is found to have a significant effect on the flow development length.

\section{Acknowledgements}

The authors are grateful to EPSRC (grant reference GR/S82978/01) for supporting this research. Additional support was provided by the EPSRC under the auspices of Collaborative Computational Project 12 (CCP12).

\section{References}

1. D. J. Harrison, A. Manz, Z. Fan, H. Lüdi, and H. M. Widmer, Anal. Chem. 64, 1926 (1992).

2. D. J. Harrison, K. Fluri, K. Seiler, Z. Fan, C. S. Effenhauser, and A. Manz, Science 261, 895 (1993).

3. A. G. Hadd, D. E. Raymond, J. W. Halliwell, S. C. Jacobson, and J. M. Ramsey, Anal. Chem. 69, 3407 (1997).

4. A. E. Herr, J. I. Molho, J. G. Santiago, M. G. Mungal, T. W. Kenny, and M. G. Garguilo, Anal. Chem. 72, 1053 (2000).

5. Y. H. Zhang, R. W. Barber, and D. R. Emerson, Proceedings of the $13^{\text {th }}$ Micromechanics Europe Workshop, 153 (2002).

6. Y. H. Zhang, X. J. Gu, R. W. Barber, and D. R. Emerson, J. Colloid Interface Sci. 275, 670 (2004).

7. $\quad$ N. A. Patankar and H. H. Hu, Anal. Chem. 70, 1870 (1998).

8. R. F. Probstein, Physicochemical Hydrodynamics: An Introduction (2 ${ }^{\text {nd }}$ ed.), (John Wiley \& Sons, New York, 1994).

9. CFD Research Corporation, Cummings Research Park, 215 Wynn Drive, Huntsville, AL 35805, USA, CFD-ACE+ User Manual: Version 2004.

10. R. J. Yang, T. I. Tseng, and C. C. Chang, J. Micromech. Microeng. 15, 254 (2005).

11. R. Y. Chen, Trans. ASME J. Fluids Engineering, 95, 153 (1973). 